\title{
A Picture Quality Control Framework for MPEG video over ATM
}

\author{
Ahmed Mehaoua ${ }^{1,2}$, Raouf Boutaba ${ }^{1}$ and Guy Pujolle ${ }^{2}$ \\ ${ }^{1}$ Computer Research Institute of Montréal, \\ 1801, Av. McGill College, Montréal (Qc), H3A 2N4 - CANADA - \\ e-mail : \{amehaoua, rboutaba\}@crim.ca \\ ${ }^{2}$ Lab. PRiSM, Université de Versailles, \\ 45, Av. des Etats-Unis 78000 Versailles - FRANCE - \\ e-mail :Guy.Pujolle@prism.uvsq.fr
}

\begin{abstract}
This paper addresses the problem of transmission of digital Video communications over B-ISDN and provides a solution based on a good knowledge of both video system design and broadband networks capabilities. We propose a picture-oriented QoS control framework to ensure a high endto-end Quality of Picture (QoP) level for MPEG-encoded Video-On-Demand (VOD) applications carried over ATM networks. Our approach overcomes the difficulty imposed by random cell discarding due to the bursty and Variable Bit Rate transmission nature of compressed video, by using an accurate cell discrimination strategy and destination data recovery mechanisms. It is assumed that there are four hierarchical types of cell flows according to MPEG coding. Each flow has a different impact on the destination picture recovery process. Therefore, this scheme aims to minimize cell loss probability for critical data and thus, reduce the destination picture quality degradation, by adjusting the priority level of cells according to the carried data type. Despite these preventive measures, cell loss or errors may still occur. Therefore, complementary actions are cooperatively taken at the source and destination equipment (i.e., codecs), in conformity with the temporal requirements of MPEG video streams. In the one hand a fast cell-error detecting and recovery algorithm is proposed for protecting critical picture system information. In the other hand, a block-based bit interleaving schemes are designed to spatially disperse cell loss effect on reference frames. Finally, an extended AAL type 5 is presented to support such a high performance picture delivery framework.
\end{abstract}

Keywords

ATM, VBR Video, MPEG coding, Quality of Service. 


\section{INTRODUCTION}

Asynchronous Transfer Mode (ATM) technology was initially designed to permit the simultaneous carrying of various services, all supported equivalently without any knowledge of the cell content i.e. without discriminating some cells against others. Among all the services, multimedia applications and especially digital video communications should contribute as a major fraction of the overall traffic. These sources are inherently variable, asynchronous, stochastic and non-stationary.

Today, it is accepted that residential audio-visual applications, such as TV broadcasting and Video-On-Demand (VOD), will work on the basis of high-speed ATM multiplexing and switching techniques and will be necessary compressed to optimize network resources utilization (i.e., bandwidth, buffers). Therefore, several coding schemes have been candidates to be the Broadband-ISDN multimedia coding standard. These video compression algorithms are mostly based on efficient Discrete Cosine Transform (DCT) and block oriented techniques. But, they are today seriously threaten by new families of coding techniques such as region-based coding, frequency decomposition or fractale transform. Among the former family, three world-wide standards were considered for use within ATM networks : ITU H.261, ISO JPEG (Joint Picture Expert Group) and ISO MPEG-1 (Moving Picture Expert Group). Unfortunately, they all have been designed to address the needs of specific services and applications, and they can not interoperate or run without restriction over ATM-based Broadband networks.

For instance, the H.261 scheme was initially designed to operate over specific digital networks (e.g. Narrowband-ISDN) with low bit rates about nx64 Kbit/s to carry teleconferencing applications where motion is naturally more limited. Similarly, JPEG algorithm only emphasizes still images and do not apply any inter-coding algorithm to take into account temporal redundancy of moving picture sequences. Finally, processing delay was not a major concern for MPEG first phase (MPEG-1), as it was not expected for use in conversational services but adapted for storage and retrieval applications of moving pictures from CDs in a loss-less environment [1].

Consequently, both ITU SG15 and ISO MPEG are collaborating to produce new higher quality video coding standards for B-ISDN, which are respectively named ISO MPEG-2 and ITU H.262 [2]. For accelerating the availability of native ATM multimedia services, the ATM Forum SAA working group has proposed the mapping of MPEG-2 transport packets into AAL5 PDU [3], and is also currently addressing the specification of a new dedicated AAL6 for lowest bit rates MPEG-4 coded applications. MPEG-4 scheduled for 1998, is designed for a whole spectrum of very low bit rates applications. These video applications will have sampling dimension up to 176 x 144 x $10 \mathrm{~Hz}$ and coded bit rates between 4800 and 64,000 bit/s (e.g. video-phony, mobile audiovisual communication, or multimedia electronic mail).

However, the use of such compression algorithms will change the flow transmission characteristic, from CBR to VBR. This will inherently cause a higher cell loss probability, a greater cell delay variation and will increase network management complexity. Since cell loss is a major problem for compressed data streams, defensive measures has to be performed at the edge (i.e., codecs) and into the network (i.e., switches) to maintain a minimum display picture quality. Despite of this demand, few QoS control schemes have been proposed to take into account the specific characteristics of bursty MPEG-encoded video streams. Most of today's traffic control researches emphasize ABR and UBR "Best effort" services, as defined in ATM Forum specifications [4]. 
Therefore, we propose a video-oriented QoS control framework for bursty video services, which is based on the layered data structure of MPEG streams. The framework allows the design of a multi-level priority policy coupled with fast data-loss recovery mechanism targeted for either system control and picture information. This approach allows the network control functions (Usage Parameter Control, Priority Control, Resource Management, ...) to discriminately react in case of congestion by preserving critical data from discarding, and performing picture concealment at the destination terminal in case of missing data.

This paper is organized as follows. We briefly review in section 2 , the main factors affecting the Quality of Picture of compressed video applications. The third section is devoted to our multi-level priority strategy and the description of the different components of the QoS control framework to guarantee a low picture quality degradation in both situations of data loss and bit-errors. A particular focus is made on network internal policies (i.e., multi-level cell priority and intelligent discarding functions) as well as edge proactive and reactive actions (resp. error recovery scheme and picture concealment through block interleaving). Finally, concluding remarks are given in section 4.

\section{FACTORS AFFECTING PICTURE QUALITY}

From a network control standpoint, applications and underlying generated bit flows are commonly grouped in two service classes according to their traffic characteristics and sensitivity. The first class is related to error and loss-sensitive flows like traditional LAN traffics. The second class concerns delay sensitive applications, which are usually referred to as real-time services (voice, non compressed video, ...).

With real-time encoded video applications, this artificial border between reliability and stringent temporal requirements are not respected. Indeed, video applications using compression capabilities are submitted to both error-free and real-time transmission constraints. Consequently, controlling the quality of service (QoS) of such demanding applications is widely considered as one of the most important challenge to be faced before introducing Broadband-ISDN multimedia services on a widespread basis.

It is thus necessary to precisely analyze the various networking factors interfering with audio and picture quality degradation. In the following, we emphasize data losses and error effects.

\subsection{Data losses due to cell errors}

Random bit errors occurring along the communication path or within the network nodes due to electrical or physical problems can highly damage the quality of the decoded pictures. At the cell level when such bit errors occur in the header, the cell is either mis-delivered when errors and address modifications are undetected, or discarded by the physical layer or by the receiver in case of uncorrectable detected errors. In both cases the whole cell should be considered as lost and the consequences can be serious for the MPEG decoding process. If the error occurs in the payload type of the cell, the damage is obviously limited to the degradation of part of the MPEG Packet. If this part belongs to the MPEG Transport Stream (TS) packet header the entire packet may be lost and the impact on the displayed pictures can be also very serious. Fortunately, the probability of 
such data losses is normally extremely low. For instance, in high-speed networks based on optical fibers, it is not exceeding $10^{-13}$. Nevertheless, even for these transient error events, new mechanisms (i.e., error detection and correction schemes) are required at the MPEG level to ensure a low video quality degradation.

\subsection{Data losses due to burstiness and excessive delays}

With regards to real-time video service, variable bit rate (VBR) transmission has several advantages over conventional constant bit rate (CBR) mode. Among the advantages, there are : consistent and subjectively better video quality, simpler encoder design and increased multiplexing gain of a factor of two and even four with cell loss probability of $10^{-6}[5][6]$. However, VBR transmission mode is an important cause of data losses due to peaks in traffic and subsequent switch buffers overflowing. These heavy loads are mostly due to inadequate network resources allocation and multiplexing processes. Exceeding network capacity leads to the cell discarding by either the congested nodes (through UPC) or the destination terminal if the delay exceeds a threshold. In the latter case, the MPEG packet arrives too late for playback on the terminal. Both cases leads to a loss of data in units of the MPEG Transport packets, which is unacceptable. Preventive actions must then be applied to minimize the QoS degradation, inside the network through intelligent discarding and, at terminal nodes, through fast data recovery schemes at both ATM and MPEG transport levels.

\section{A PICTURE QUALITY CONTROL FRAMEWORK}

MPEG scheme handles three frame types which are distinguished by the used compression method : Intra-coded (I frames), Predictive coded (P frames) and Bi-directional predictive coded (B frames). I frames are encoded using only a spatial DCT compression and do not refer to any other frames. P frames use DCT and a simple Motion Compensation (MC) with the reference to the previous I or P frames. Finally, B frames use DCT and MC Interpolation with respect to the previous I or $\mathrm{P}$ frames and the next I or P frames. In order to increase coding efficiency, a typical arrangement is composed with periodic interleaving of frames from each mode. This deterministic periodic sequence is called Group Of Pictures (GOP) and depicted by Figure 1.

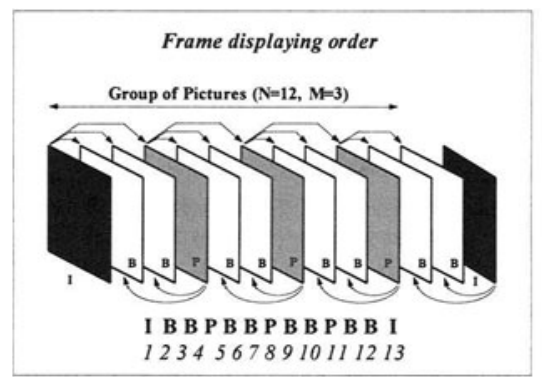

Figure 1 - Group Of Pictures (GOP)

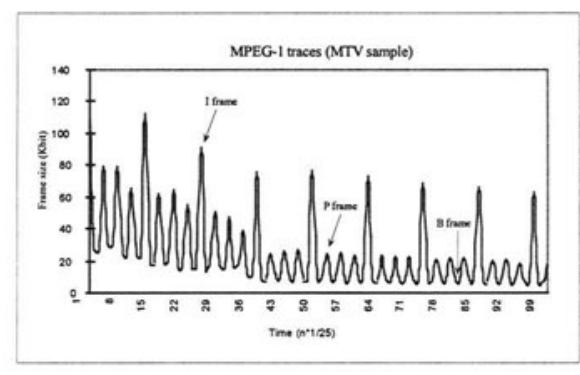

Figure 2 - Impact of coding modes on frame size. 
Regarding to their importance for the decoding process and thus on the displayed picture quality, I frames have to be send with a guaranteed delivery service. Similarly, clock references and system data, located at the TS and Packetized Elementary Stream (PES) headers, have to be protected from loss or errors to allow the destination system controller to segregate and synchronize the audio and video sub-flows. Assuming this priority hierarchy between MPEG data, it is our goal to propose a video-oriented approach for traffic management and congestion control operations to better take into account the delay and loss sensitive characteristics of MPEG-encoded applications. Therefore, dedicated proactive and reactive QoP control policies are described in the following sections.

\subsection{Proactive Network Control policies}

\subsubsection{Protection by shaping and policing targeted for encoded video streams}

The call admission control (CAC) function is not sufficient to prevent cell discarding and upperlayer MPEG packet loss. Indeed, users may not respect the connection parameters negotiated at the call set-up (Average Cell Rate, Peak Cell Rate, Sustainable Cell Rate, Maximum Burst Size, ...). Network operators must then, ensure that the sources stay within their connection parameters. These functions, referred to as traffic shaping and traffic policing, should detect non- conforming cells generation and set appropriate actions to minimize the negative effect of traffic excess. Several actions are proposed in ITU-T I.371 recommendation [7], dropping, delaying, or marking the non-conforming cell and/or notifying the violating source to reduce its bit rate (Explicit Backward Congestion Notification).

Due to the bursty characteristics of encoded video streams, the buffering of cells before they enter the network (i.e., shaping) will significantly reduce bandwidth utilization but may extend delays to unacceptable limits. For instance, at the start of a Video-on-Demand session, a fast load is performed of the first few minutes of a movie from the selected Video Server. This action requires a large bandwidth, e.g., $100-150 \mathrm{Mb} / \mathrm{s}$, to be allocated in the network during few seconds. This provides the user with quasi immediate access to the service, but at the same time the video signal rate of the remaining parts of the movie decreases to several $\mathrm{Mb} / \mathrm{s}$ (about 2-8 $\mathrm{Mb} / \mathrm{s}$ ). Similarly, random cell discarding (i.e., policing) can cause serious degradation of the picture quality.

It is our goal to provide a video-oriented approach for traffic management and congestion control operations to better take into account the delay and loss sensitive characteristics of MPEGencoded applications.

\subsubsection{A video-oriented priority scheme and an associated discarding policy}

As discussed previously, indiscriminate cells dropping leads to a visible damage at pictures display. This can be overcame by use of an effective four-state priority scheme associated with an intelligent discarding strategy and based on MPEG multi-level data structure.

To avoid a congestion worsening or an excessive jitter, a lower priority cell, in the MPEG coding sense, is dropped (rather than delayed) and its buffer space is given to a higher priority cell. For our purpose, we focus on a shared buffer approach. The results in [8] indicate that the performances are increased but at the cost of a higher complexity of an ATM switch management. 
A multi-level priority strategy, named Early Packet Discarding (EPD), has been previously proposed for discarding a set of cells. For packet-oriented services when an intermediate node discards a cell, all the following cells associated with the same packet have no use to be transmitted anymore and can be removed. In our case, we adapt such a capability to discriminate the cells belonging to the different MPEG frame types (I, P, B, System control) and which are candidate for discarding. During congestion, groups of B-cells between either P or Icells are firstly dropped. This will statistically reduce overload buffers occupancy by a ratio of about $13 \%$. This allows network control and management functions to ensure a graceful picture quality degradation for MPEG connections. We should notice that EPD mechanism, in relation with AAL5 protocols, uses a marked bit belonging to the PTI field to determinate ends of data packets.

To implement priority management capabilities, ITU-T has proposed the use of the one-bit CLP mechanism to distinguish between two types of cell within a VC stream. Actually, three types of priority cells can be defined according to the value of the CLP bit : CLP $=0$ for high priority cells, $\mathrm{CLP}=1$ for low priority cells and $\mathrm{CLP}=0+1$ for tagged cells.

Based on this analysis, we are able to define two types of priority levels in ATM networks: a "service-oriented" priority and a "congestion-oriented" one. The former reflects the relative importance of one cell over another (user point of view), while the latter is generally assumed by the UPC/NPC mechanisms (traffic control point of view). According to the ATM cell structure a conflict arises when the CLP bit is used to implement these both levels of priority assignments. Consequently, there is no possible distinction between the service-oriented (vital vs. non-vital) and congestion-oriented (conformed vs. non-conformed) markings of cells [9].

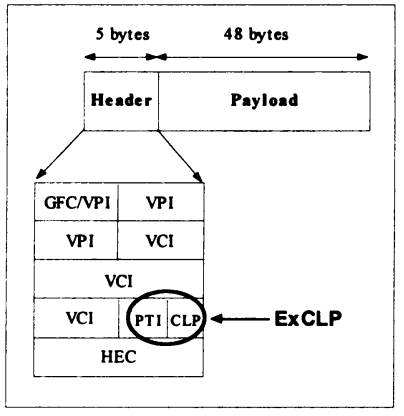

\begin{tabular}{|c|c|c|c|}
\hline Data Type & $\begin{array}{c}\text { CLP } \\
\text { bit }\end{array}$ & $\begin{array}{c}\text { PT } \\
\text { bit }\end{array}$ & $\begin{array}{c}\text { Priority } \\
\text { level }\end{array}$ \\
\hline System_data & 0 & 0 & Very High \\
\hline I frames & 0 & 1 & High \\
\hline P frames & 1 & 0 & Low \\
\hline B frames & 1 & 1 & Very Low \\
\hline
\end{tabular}

Table 1 - ExCLP and MPEG data mapping

Figure 3 - ATM cell header

To overcome this difficulty, our control scheme applies a four-state priority strategy using two bits. The first one is the classical CLP bit and the second one is the adjacent bit belonging to the PTI field (Figure 3). This new field, referenced as Extended CLP (ExCLP), allows each CLP and PTI bit to singly assume their original meanings according to standards [10]. Four priority classes are defined and MPEG data types are mapped as shown in Table 1.

With respect to the MPEG compression algorithm, the System_data class, which regroups audio data and the following MPEG headers (Transport Stream, PES, Sequence, GOP, Frame, Slice and Audio) are considered as critical information and are assigned the highest priority level. They mostly convey audio-video synchronization data and coding control information related with 
each picture level. The loss of such data will, in the best case, generate audio or picture silences, and in the worst case, the loss of the whole MPEG TS packet. For instance, the slice_start_code field located at the slice header indicates the position of each slice in the whole picture, and obviously has to be preserved from loss. The cells carrying I-frames data should also have a high priority level, since they embed intra picture information and are referenced by other frames. The two other types of frames ( $\mathrm{P}$ and $\mathrm{B}$ ) are assigned a lower priority levels.

When a network congestion occurs, B-cells are firstly discarded, while P, I and system_data cells are preserved from elimination during network crossing. To quickly reduce buffer occupancy as well as a low QoS degradation, it is suitable to perform an Early Packet Discarding scheme on successive groups of B-cells. Statistically, B-cells represents about $13 \%$ of the whole streams for a common GOP pattern $(\mathrm{N}=12$ and $\mathrm{M}=3)$. If buffer overflowing persists, the node may further discard P-cells (29\%) and I-cells (53\%). This measures will ensure a graceful and manageable QoS degradation, by means of a selective cells discarding strategy and cooperative data recovery techniques at the source and destination equipment.

\subsubsection{A video-oriented Adaptation ATM Layer for QoS control support}

In order to realize the previous multi-level priority management of MPEG-2 bit-streams, appropriate adaptation protocols are necessary. The ATM Forum recommends the carrying of two MPEG-2 Transport Stream packets per AAL5-PDU with a NULL Convergence Sub-layer [11] (Figure 4a). This way, the Quality of Service of bursty interactive VOD applications are not guaranteed. This topic is definitely still an open issue.

Real-time video services require a more sophisticated AAL than the simple AAL type 5 standard. Therefore, we propose an extended AAL5, namely AAL5+, which provides further picture quality control facilities. Among the additional features offered by AAL5+, there are a new specific CS layer and the ability to perform two different segmentation and packetization processes on two types of CS-PDU.

The aim of the specific CS sub-layer is to allow a first-step MPEG data extraction process. The AAL5+ SSCS protocol, is composed of a one-bit header and a fixed payload. Regarding to the bit-header value, the SSCS-PDU carries MPEG-2 TS and PES headers (0) or only PES payload types (1).

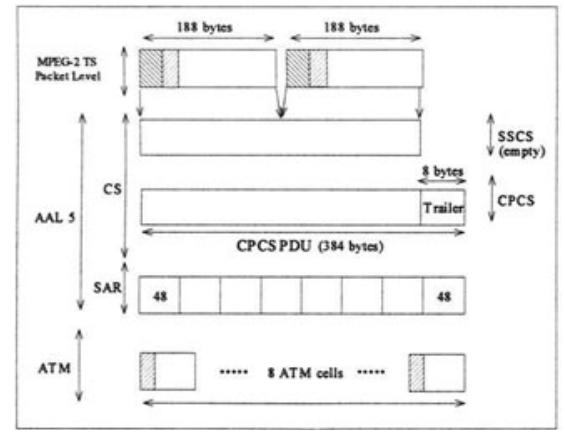

Figure 4a - Classical mapping of MPEG-2 Transport Stream packets over AAL5

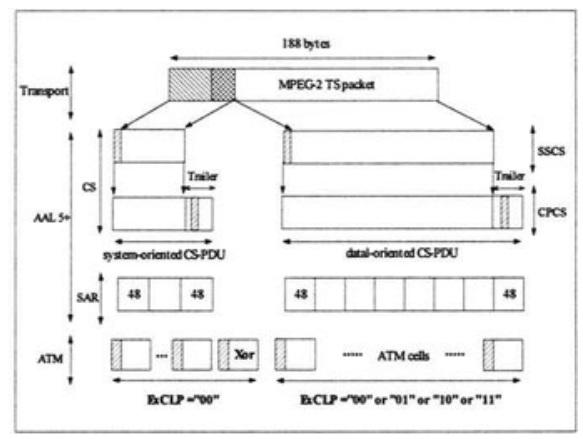

Figure 4b - New mapping of MPEG Transport Stream packet over AAL5+ 
One bit from the AAL5 CPCS-user-to-user indication field is newly used to discriminate between the SSCS-PDUs received from the upper-layers. The seven remaining (7) bits of the CPCS-UU field are used as a sequence number (SN) for our cell-loss detection and recovery scheme (see section 3.2.1). This is a light modification of the previous AAL5 standard which allows a full protocol interoperability. Moreover, the AAL 52 bytes control field is not yet used or specified by standard bodies. This modification allows the definition of two types of CS-PDU, namely System-oriented CS-PDU and Data-oriented CS-PDU (see Figure 4b).

The under-laying SAR protocol will subsequently segment each PDU into ATM cells and set the ExCLP field according to the CS-PDU type and the MPEG syntax. A segmentation process is defined for each CS-PDU type.

System-oriented PDUs are simply segmented into cells with each ExCLP field set to '00' (i.e., very high priority), whereas the Data-oriented CS-PDUs are concerned with a different segmentation and packetization process. Actually, a parsing scheme, with respect to MPEG syntax, is performed to build the following data cells. This stream parsing will extract audio data and MPEG headers from picture data. The headers and audio data are then transmitted in cells with ExCLP field set to ' 00 ', while for picture data, the ExCLP value depends on their coding mode. The current ExCLP's cell value is assigned according to the picture_coding type field located in the frame header. This picture_coding_type field specifies for each frame the used coding mode (Intra, Predictive or Bi-directional).

\subsection{Reactive destination policies}

\subsubsection{System data protection through data-loss detection and recovery scheme.}

For a cell with one corrupted bit, a Header Error Control (HEC) model is efficient, but in ATMbased broadband networks, corrupted cells are usually the result of a physical transmission problem. When errors occur, they typically take the form of long bursts. Therefore, too much data are damaged in the burst and HEC scheme is not able to correct them. Our approach is to consider corrupted cells as missing cells and then perform a cell lost recovery algorithm on critical system data information.

As depicted in Figure 5, cell loss can be corrected by structuring a set of AAL5+ PDU as a square block and padding an AAL5+ PDU embedding parity-check cells performed on each column. At the destination system, this can be seen as a virtual $(\mathrm{N}+1)^{*}(\mathrm{M}+1)$ cell matrix. Whereas at the source system, it is only an $(\mathrm{N}+1)^{*} \mathrm{M}$ cell matrix, with the $(\mathrm{N}+1)$ row of cells containing parity bits for checking the corresponding bits in the associated column direction. Since the source systems are able to insert a sequence number with our AAL5+ layer (using the new added 7 bits), cell loss can be detected and the position of the lost cell(s) easily located. The contents of the parity cell in the $(\mathrm{N}+1)$ row can then be used to restore the lost cell(s). At this step, no more than one cell per column can be recovered. Thus, in case of serious congestion transition, we proposed to process two additional parity-check cells in relation with each diagonal of the matrix. According to [12], this extended mode allows us to recover up to two lost cells per column.

For bandwidth saving and fast processing considerations, we rather use the first mode. But if necessary, the second mode can be activated at reception of a notification message from the network (switches or destination equipment), or from a higher management entity. This scheme has also a useful feature in that it allows the parity-check cells to be computed while data is being 
transmitted. This way, there is no need to buffer cells before transmission. But if a cell is lost and detected by the AAL5 CRC, the receiver will have to buffer the whole set of cells for the recovery process.

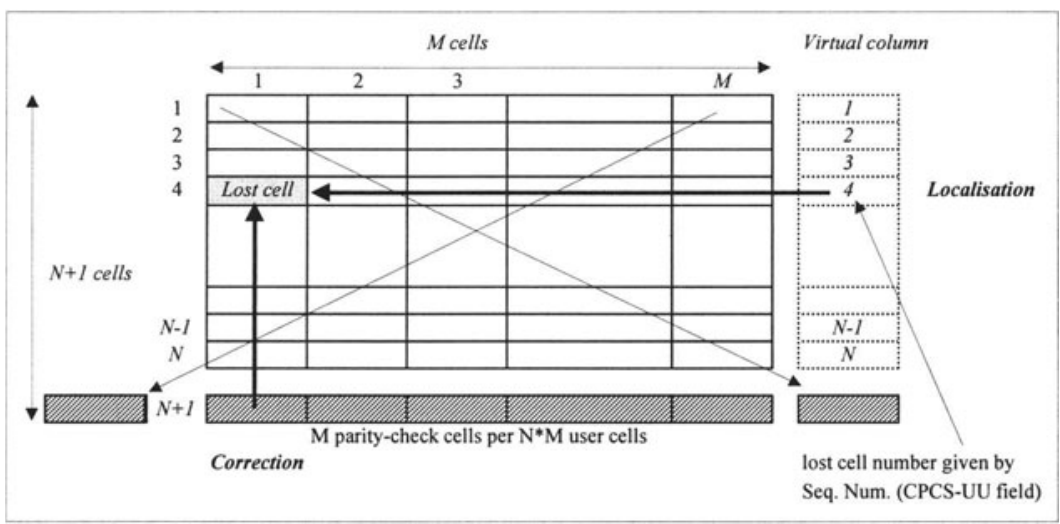

Figure 5 - Enhanced cell loss detection and recovery scheme.

Due to the GOP pattern $(\mathrm{N}=12, \mathrm{M}=3)$, I-frames are generated every 12 frames and approximately correspond to $50 \%$ of the whole data amount. P and B frames respectively represent 30 and $15 \%$. To reduce control overhead and processing time, this data recovery process is only performed on System_data cells (about 5\%). If missing or erroneous I-cells are detected, a block-based bit interleaving scheme is performed at the destination to reduce errors effects on local picture sections.

\subsubsection{I-frames protection by Block Interleaving}

In the MPEG video coding scheme, adjacent data are highly correlated. This high degree of correlation can be used to hide any missing picture block by using data from adjacent or closest possible blocks. This technique is only effective if adjacent coded data are interleaved in such a way that encoded data for neighboring block regions are placed in separated transport data units (i.e., ATM cells).

We use such a strategy at the SAR protocol level to protect referenced Intra-coded frames from random block losses. The cell segmentation and packetizing would then proceed not according to a traditional scanning order, but in an interleaved order. This way the effect of an Icell loss, at the receiver, will be spatially dispersed over a larger picture section and will enhance displayed picture quality.

With consumer High-Definition TV standard (1440x1152x30fps), each MPEG-2 block section is composed of $8 * 8$ picture elements (pixels) with chromatic and luminance resolutions of 24-bits (i.e., high color format). Each MPEG-2 block is then transported in exactly four (4) ATM cells $(8 * 8 * 24=1536$ bits $=192$ bytes $=4 * 48$ ATM payload types). Using this property, we define an efficient bit interleaving process on MPEG-2 block and ATM cell basis illustrated by Figure 7 . 


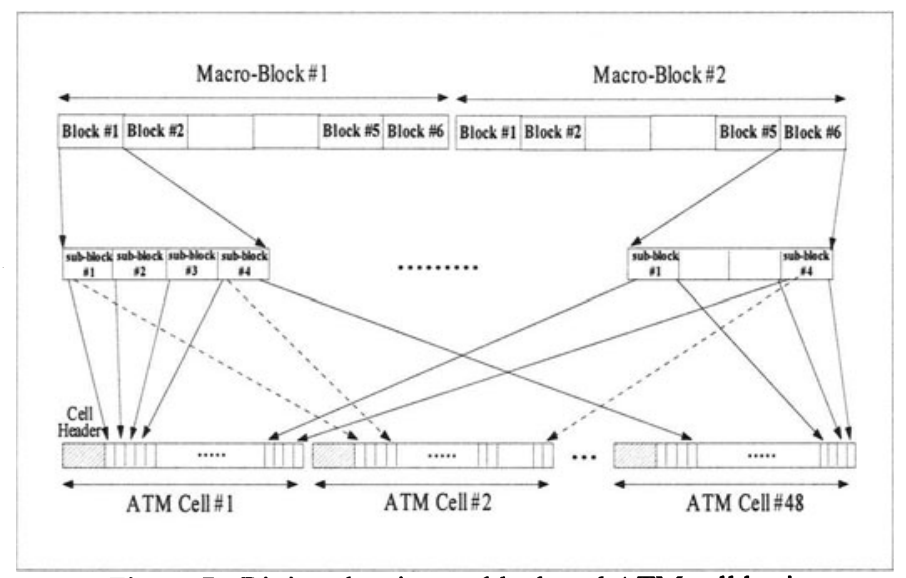

Figure 7 - Bit interleaving on block and ATM cell basis

\section{CONCLUSION}

In this paper, we have surveyed a number of topics related to the coding and control of audiovisual on-demand applications conveyed over ATM networks. A particular attention has been given to the MPEG-2 compression scheme and the associated transport data structure, since it is adopted as the coding standard for deploying multimedia services over Broadband-ISDN. More specifically, we have analyzed the network factors affecting the Quality of Service of MPEGencoded VOD applications and we have shown the need of dedicated video-oriented QoS control policies for such a service.

In this perspective, the framework presented in this paper has been designed to allow a refined picture quality control based on MPEG-2 data structure and characteristics. The framework implements in a harmonized way proactive and reactive control policies at both the network edge (i.e., codecs) and within the network (i.e., switches). The proactive policies allow to protect highly sensitive data through a four-level priorities assignment. The reactive policies are performed at the destination equipment to recover cell loss and errors. By introducing semantic information at the transmission level (i.e., ATM cell level) concerning types of the data carried by the cells, we have shown how the proposed framework together with the defined enhanced Adaptation ATM Layer type 5 allow to discriminately and efficiently deal with network congestion.

The ultimate aim of this work has been to ensure graceful end-to-end picture quality degradation for current video applications over broadband networks. For demanding real-time compressed video service which is characterized by a high congestion probability, we believe that our dedicated framework will bring a better quality. The overhead introduced by the complexity of the presented control policies will be lightened by the processing power available in today's multimedia end equipment and by off-line processing capability. For instance, Video-on-Demand servers have an easier time to store all the programs as ATM cells with priority assignment rather 
than just bit streams [13]. Finally, we are now planning to experiment our approach and the implemented algorithms on an ATM testbed that is currently being deployed.

\section{REFERENCES}

[1] ISO/IEC DIS 2-11172 MPEG-1, "Information Technology - Coding of moving pictures and associated audio for digital storage media up to about $1.5 \mathrm{Mbit} / \mathrm{s}$ ", 1993 .

[2] ISO/IEC 13818-2 MPEG-2-H.262, "Information Technology - Generic Coding of Moving Pictures and Associated audio", Committee draft, UK, March 1994.

[3] ATM Forum SAA SWG, "Audiovisual Multimedia Services : Video on Demand", Specification 1.0, ATM_FORUM/af-saa-0049.000, December 1995.

[4] ATM Forum TM SWG, "Traffic Management Specification 4.0", ATM-Forum/950013R8, October 1995.

[5] W. Verbiest, L. Pinno, and B. Voeten, "The impact of the ATM Concept on Video Coding", IEEE JSAC, vol.6, n, pp1623-1632, December 1988.

[6] D. Heyman, A. Tabatabai, and T. V. Lakshman, "Statistical Analysis and simulation Study of Video Teleconference Traffic in ATM Networks", IEEE JSAC, vol.2, $\mathrm{n}^{\circ} 1$, pp. 49-59, March 1992.

[7] ITU-T I.371, "Traffic Management and Congestion Control in B-ISDN", March 1993.

[8] J.W. Causey and H.S. Kim, "Comparison of Buffer Allocation Schemes in ATM Switches : Complete Sharing, Partial Sharing, and Dedicated Allocation", IEEE 1994.

[9] A. Gravey, and al., "Tagged Versus Strict Rate Enforcement in ATM Networks," IEEE GLOBECOM'91, Phoenix, pp. 218-288, 1991.

[10] ITU-T I.361 specification of ATM layer, March 1993.

[11] J. Zhang, and al., "Carrying MPEG-2 Transport Streams using AAL5", ATMForum/94-0879, Sept. 1994.

[12] N. Shacham, P. McKenney, "Packet Recovery in high-speed networks using coding and buffer management", IEEE INFOCOM'90, June 1990.

[13] Y. Chang, D. Coggins, and al., "An Open-systems Approach to Video on Demand", IEEE Communication Magazine, vol. 32, n5, pp. 68-80, May 1994. 